\section{Commentary: Simplified innominate artery antegrade cerebral perfusion}

\author{
Chris Burke, MD, and Gabriel Aldea, MD
}

The incidence of neurologic dysfunction following arch surgery remains significant $(6 \%-16 \%)$ and varies with the extent, complexity, and acuity of the underlying pathology. ${ }^{1-5}$ Protective strategies to mitigate neurologic dysfunction following arch surgery continue to evolve and include systemic hypothermia, typically supplemented with retrograde and/or antegrade cerebral perfusion (ACP; unilateral, bilateral, axillary/subclavian or innominate arteries). Results (mortality and permanent neurologic deficits) are comparable between cerebral perfusion strategies, with a trend toward lower transient neurologic deficits and intensive care unit stays with ACP. ${ }^{4}$ ACP is recommended by current guidelines ${ }^{6,7}$ and is the workhouse cerebral-protection strategy for complex arch reconstructions. Axillary artery cannulation, typically using a graft sewn in an end-to-side fashion, serves as a convenient and useful way to provide both systemic perfusion and ACP. However, concerns exist over the small but significant risk of vessel injury, brachial plexopathy, or pectoral muscle atrophy. ${ }^{2}$ This has led to increasing enthusiasm for innominate artery cannulation for ACP delivery as an alternative to axillary cannulation.

Sang and colleagues ${ }^{8}$ present their technique and results of a simplified technique of direct innominate artery cannulation with a 12/14-French pediatric arterial cannula used during elective non-redo hemiarch replacements,

From the Department of Cardiothoracic Surgery, University of Washington, Seattle, Wash.

Disclosures: The authors reported no conflicts of interest.

The Journal policy requires editors and reviewers to disclose conflicts of interest and to decline handling or reviewing manuscripts for which they may have a conflict of interest. The editors and reviewers of this article have no conflicts of interest.

Received for publication March 12, 2020; revisions received March 12, 2020; accepted for publication March 13, 2020; available ahead of print April 3, 2020.

Address for reprints: Gabriel Aldea, MD, Division of Cardiothoracic Surgery, University of Washington, Room AA115, Box 356310, 1959 NE Pacific St, Seattle, WA 98195-6310 (E-mail: aldea@uw.edu).

JTCVS Techniques 2020;2:16-7

2666-2507

Copyright $(2020$ The Authors. Published by Elsevier Inc. on behalf of The American Association for Thoracic Surgery. This is an open access article under the CC BY-NCND license (http://creativecommons.org/licenses/by-nc-nd/4.0/).

https://doi.org/10.1016/j.xjtc.2020.03.013

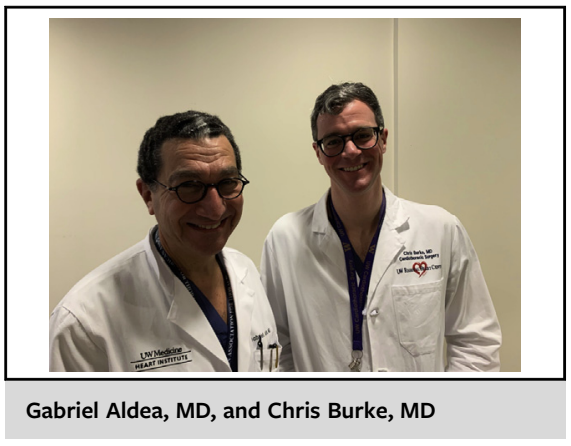

CENTRAL MESSAGE

Direct innominate artery cannu-

lation is a safe and effective tool

to deliver ACP during aortic arch

reconstruction and should be

incorporated as part of comple-

mentary neuroprotective

strategies.

demonstrating safety and efficacy. This technique avoids the added length, complexity, and complications associated with axillary artery exposure, addresses ACP, but requires supplementation with central cannulation to accomplish systemic flows. The authors certainly demonstrate the safety of this technique in a low-risk population, as no innominate artery injuries were noted. However, the fact remains that in straightforward hemiarch reconstructions, given circulatory arrest times are typically at or below 20 minutes, there exist a multitude of cerebral-protection strategies that are safe and effective, including retrograde cerebral perfusion techniques. It is in the more complex aortic arch procedures that this protection strategy will likely enjoy the most utility, including in transverse arch reconstruction and frozen elephant trunks, as an alternative to axillary cannulation or direct cerebral vessel cannulation. Further, some groups have advocated for innominate (or carotid) cannulation during acute type A dissection repair with cerebral malperfusion. ${ }^{9}$

It is critical for the aortic surgeon to be facile with multiple cerebral-protection strategies and avoid a "one-sizefits-all approach." These therapies are complementary, and their use (of one or multiple strategies) needs to be individualized to meet an anatomical, aortic pathology, and 
patient-specific needs and enhance outcomes. Direct innominate artery cannulation is a safe and effective tool for deliver ACP during aortic arch reconstruction, and its utility will continue to evolve.

\section{References}

1. Takagi H, Mitta S, Ando T. A contemporary analysis of antegrade versus retrograde cerebral perfusion for thoracic aortic surgery. Thorac Cardiovasc Surg. 2018;67:351-62.

2. Preventza O, Price MD, Spiliotopoulus K, Amarasekara HS, Cornwell LD, Omer S, et al. In elective arch surgery with circulatory arrest, does arterial cannulation site matter? A propensity score analysis of right axillary and innominate artery cannulation. J Thorac Cardiovasc Surg. 2018;155:1953-60.

3. De Paulis R, Czerny M, Weltert L, Bavaria J, Borger MA, Carrel TP, et al. Current trends in cannulation and neuroprotection during surgery of the aortic arch in Europe. Eur J Cardiothorac Surg. 2015;47:917-23.

4. Okita Y, Miyata H, Takamoto N, Takamoto S; Japan Cardiovascular Surgery Database Organization. A study of brain protection during total arch replacement comparing antegrade cerebral perfusion versus hypothermic circulatory arrest, with or without retrograde cerebral perfusion: analysis based on the Japan adult cardiovascular surgery database. J Thorac Cardiovasc Surg. 2015;149: S65-73.

5. Hsieh WC, Kan CD, Yu HC, Aboud A, Lindner J, Henry BM, et al. Ascending aortic versus total arch replacement in the treatment of acute type A dissection: a meta analysis. Eur Rev Med Pharm Sci. 2019;23:9590-611.

6. Erbel R, Aboyans V, Boileau C, Bossone E, Bartolomeo RD, Eggebrecht H, et al 2014 ESC guidelines on the diagnosis and treatment of aortic diseases: document covering acute and chronic aortic diseases of the thoracic and abdominal aorta of the adult. The task force for the diagnosis and treatment of aortic diseases of the European Society of Cardiology (ESC). Eur Heart J. 2014;35:2873-926.

7. Svensson LG, Adams DH, Bonow RO, Kouchoukos NT, Miller DC, O'Gara PT, et al. Aortic valve and ascending aorta guidelines for management and quality measures: executive summary. Ann Thorac Surg. 2013;95:1491-505.

8. Sang SLW, Beute TJ, Timek T. A simple method to establish antegrade cerebra perfusion during hemiarch reconstruction. J Thorac Cardiovasc Surg Tech. 2020;2:10-5.

9. Rylski B, Urbanski PP, Siepe M, Beyersdorf F, Bachet J, Gleason TG, et al. Operative techniques in patients with type A dissection complicated by cerebral malperfusion. Eur J Cardiothorac Surg. 2014;46:156-66. 